\title{
Routing and Wavelength Assignment in Optical Networks ${ }^{1}$
}

\author{
by
}

\section{Asuman E. Ozdaglar and Dimitri P. Bertsekas ${ }^{2}$}

\begin{abstract}
The problem of routing and wavelength assignment (RWA) is critically important for increasing the efficiency of wavelength-routed all-optical networks. Given the physical network structure and the required connections, the RWA problem is to select a suitable path and wavelength among the many possible choices for each connection so that no two paths sharing a link are assigned the same wavelength. In work to date, this problem has been formulated as a difficult integer programming problem that does not lend itself to efficient solution or insightful analysis. In this work, we propose several novel optimization problem formulations that offer the promise of radical improvements over the existing methods. We adopt a (quasi-)static view of the problem and propose new integerlinear programming formulations, which can be addressed with highly efficient linear (not integer) programming methods and yield optimal or near-optimal RWA policies. The fact that this is possible is surprising, and is the starting point for new and greatly improved methods for RWA. Aside from its intrinsic value, the quasi-static solution method can form the basis for suboptimal solution methods for the stochastic/dynamic settings.
\end{abstract}

\section{INTRODUCTION}

Optical networks employing wavelength division multiplexing (WDM) offer the promise of meeting the high bandwidth requirements of emerging communication applications, by dividing the huge transmission bandwidth of an optical fiber ( $\sim 50$ terabits per second) into multiple communication channels with bandwidths $(\sim 10$ gigabits per second) compatible with the electronic processing speeds of the end users.

There has been great interest in WDM networks consisting of wavelength routing nodes interconnected by optical fibers. Such networks carry data between access stations in the optical domain without any intermediate optical to/from electronic conversion. To be able to send data from one access node to another, one needs to establish a connection in the optical layer similar to the one in a circuit-switched network. This can be realized by determining a path in the network between the two nodes and allocating a free wavelength on all of the links on the path. Such an all-optical path is commonly referred to as a lightpath and may span multiple fiber links without any intermediate electronic processing, while using one WDM channel per link. The entire bandwidth on the lightpath is reserved for this connection until it is terminated, at which time the associated wavelengths become available on

1 Research supported in part by Grant ONR N00014-99-1-1019.

2 Dept. of Electrical Engineering and Computer Science, M.I.T., Cambridge, Mass., 02139. 
all the links along the route.

In the absence of wavelength conversion, it is required that the lightpath occupy the same wavelength on all fiber links it uses. This requirement is referred to as the wavelength continuity constraint. However, this may result in the inefficient utilization of WDM channels. Alternatively, the routing nodes may have limited or full conversion capability, whereby it is possible to convert an input wavelength to a subset of the available wavelengths in the network.

Since lightpaths are the basic building block of this network architecture, their effective establishment is crucial. It is thus important to provide routes to the lightpath requests and to assign wavelengths on each of the links along this route among the possible choices so as to optimize a certain performance metric. This is known as the routing and wavelength assignment (RWA) problem. The wavelengths assigned must be such that no two lightpaths that share a physical link use the same wavelength on that link. Moreover, in networks without wavelength converters, the same wavelength must be used on all links of the lightpath (wavelength continuity constraint). The RWA problem is critically important in increasing the efficiency of wavelength-routed optical networks. With a good solution of this problem, more customers can be accommodated by the given system, and fewer customers need to be rejected during periods of congestion.

Numerous research studies have been conducted on the RWA problem. Several RWA schemes have been proposed that differ in the assumptions on the traffic pattern, availability of the wavelength converters, and desired objectives. The traffic assumptions generally fall into one of two categories: static or dynamic. In static RWA models we assume that the demand is fixed and known, i.e., all the lightpaths that are to be set up in the network are known beforehand. The objective is typically to accommodate the demand while minimizing the number of wavelengths used on all links. By contrast, in a stochastic/dynamic setting, we assume that lightpath requests between source-destination pairs arrive one by one at random, and have random terminating times. A typical objective in this case would be to minimize the call blocking probability, or the total (perhaps weighted) number of blocked calls over a given period of time.

Even in the simpler static case, typical proposed formulations for optimal lightpath establishment turn out to be difficult mixed integer linear programs. In particular, the optimal static lightpath establishment problem without wavelength converters was proven to be NP-complete in [CGK92] by showing the equivalence of the problem to the graph-coloring problem. Since the associated integer linear programs are very hard to solve, the corresponding relaxed linear programs have been used to get bounds on the desired objective function [RaS95]. Alternative formulations have been considered to get tighter bounds. These bounds are used as benchmarks against which performance of various heuristic RWA algorithms can be compared.

Due to computational complexity in obtaining an optimal solution, much of the previous work on the routing and wavelength assignment problem has focused on developing efficient heuristic methods. A common approach is to decouple the routing and wavelength assignment steps by first finding a route from a predetermined set of candidate paths and then search for an appropriate wavelength assignment [BaM96], [SuB97]. However, given that the number of wavelengths is restricted, a common wavelength may not be available on all the links along a chosen route. Thus routing and wavelength assignment should be considered jointly for best performance. 
A lot of recent work in WDM networks is based on the maximum-load model [GSKR99], [GeK97], [RaSi98]. In this model, the route of each request is given and the problem is to find the minimum number of wavelengths to satisfy a given request set. This is a worst-case model, where no blocking of lightpaths is allowed, and there are no assumptions made on the traffic pattern. The traffic is characterized only by its load, which is the maximum number of lightpaths that can be present over any link in the network. However, this results in overdesigning the network and using many wavelengths to support atypical request patterns.

Most of the literature on the RWA problem considers either networks without any wavelength converters or networks with wavelength converters at every node. The benefits of wavelength conversion have been analyzed under different traffic models [BaH96], [GSKR99]. However, the high cost of full wavelength conversion at every node has led to some research on networks with sparse wavelength conversion [SAS96], [GSKR99], [RaS98]. In such a network, only a fraction of the nodes are equipped with wavelength converters. We assume that we have full wavelength conversion capability at the nodes where there are wavelength converters. The blocking performance of such a network has been analyzed in [SAS96] assuming a statistical traffic model and a simple routing-wavelength allocation scheme.

There has also been considerable interest in obtaining the call blocking performance of wavelength-routed networks under dynamic traffic assumptions [BaH96], [KoA96], [SaS96]. For this purpose, stochastic models are employed for the call arrivals and service times. The performance of the network is studied when some simple routing-wavelength allocation methods are used. The main goal in these studies is to identify important network parameters that affect the blocking performance of the network.

In this paper, we develop an efficient algorithmic approach for optimal routing and wavelength assignment for optical networks. Our approach can be used for networks with no wavelength conversion and easily extends to networks with sparse wavelength conversion. In a general formulation, we may consider a dynamic and stochastically varying demand model, where it is important that present-time decisions take into account the effect of the uncertain future demand and availability of resources. This formulation leads to dynamic programming problems, which are difficult to solve optimally. We therefore propose in Section 2 a simpler quasi-static view of the problem, based on optimal multicommodity routing, which is closer to the currently existing approaches. However, we take into account the effect of the present-time decisions on future resource availability by means of a cost function which tends to leave room for future lightpath establishments.

The key new aspect of our formulation that sets it apart from other approaches, is that mainly because of the structure of the cost function, the resulting formulation tends to have an integer optimal solution even when the integrality constraints are relaxed, thereby allowing the problem to be solved optimally by fast and highly efficient linear (not integer) programming methods. Because of the optimality of the solutions produced, our methodology is not subject to the performance degradation that is inherent in the alternative heuristic approaches. We prove the optimality of resulting solutions in Section 3 for special but widely used in practice topologies, such as ring networks under some assumptions. For the case when our approach fails to find an integer optimal solution for arbitrary network topologies that has full wavelength conversion, we provide an efficient rounding method in Section 4. This method takes into account the structure of the cost function, and starting from an optimal noninteger 
solution, produces a possibly suboptimal integer solution. It may also be used to construct efficient methods that find optimal or near-optimal solutions for the no wavelength conversion case. However, based on our ring network analysis, as well as extensive computational experimentation, it is likely that an integer optimal solution can be found by our methodology for most optical networks and traffic patterns encountered in practice.

\section{A LINEAR PROGRAMMING APPROACH}

We propose to address the routing and wavelength assignment problems jointly for an optical network. At the most general level, we consider the scenario illustrated in Fig. 1. At first, we are given a set of lightpath requests to be established (static traffic). Then additional lightpath requests arrive randomly, one at a time. Lightpath requests are terminated randomly as well. We would like to assign routes and wavelengths to the new requests without rerouting the existing lightpaths. However, since the number of wavelengths is limited, statistically, some of the lightpath requests will be blocked. Assuming that there is a cost associated with blocking a lightpath, a reasonable goal is to minimize the expected value of the sum of the blocking costs. This problem is a dynamic programming problem, which models the stochastic nature of future lightpath arrivals/departures and incorporates this information into each routing and wavelength assignment decision.

Static Traffic Dynamic Traffic

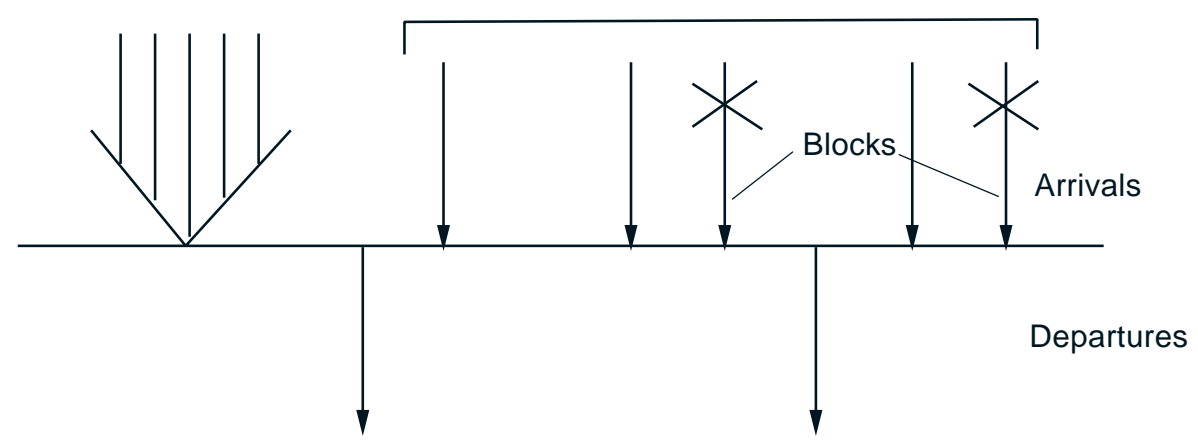

Figure 1. Conceptual view of a dynamic/stochastic model. A set of lightpath requests to be established is initially given (static traffic). Additional lightpath requests arrive randomly over time, and lightpath requests are also terminated randomly. If, due to statistical fluctuations, the network becomes congested, some of the lightpath requests cannot be accommodated and must be blocked, thereby incurring blocking costs.

The optimization/decision variables of the dynamic programming problem are the routing and wavelength assignment (RWA) decisions to be made each time there is a new lightpath request. The state of the system is the set of established lightpath requests plus the new lightpath request, and the new state is changed based on the corresponding RWA decision. A cost is incurred each time a lightpath request must be blocked due to wavelength unavailability. Unfortunately, the state space for this dynamic programming problem is prohibitively large. While it is possible to address the computational difficulty by approximations, in this paper we adopt a different approach. Although more static in character, this approach still addresses to some extent the dynamic nature of the problem by spreading the traffic in such a way that no link is operated close to its capacity. 
Our new approach is a type of optimal multicommodity flow formulation. Multicommodity network flow problems involve several flow types or 'commodities', which simultaneously use the network and are coupled through either link capacities or through the cost function. At the most general level, the optimal multicommodity flow formulation takes the form

$$
\begin{aligned}
\text { minimize } & \sum_{l \in L} D_{l}\left(f_{l}\right) \\
\text { subject to } & \text { conservation of flow constraints } \\
& \text { plus any additional special constraints, }
\end{aligned}
$$

where $f_{l}$ denotes the total flow on link $l$, and $L$ is the set of links in the network. The link cost function $D_{l}$ is typically chosen to be a convex monotonically increasing function. As a result, this formulation tends to spread the traffic and keep the link flows away from link capacity, thereby resulting in efficient bandwidth utilization and minimizing blocking of new traffic.

In the context of optical networks, different commodities correspond to different lightpaths to be established between nodes of the network. Let us first focus on the simple case where we have full wavelength conversion at all the routing nodes. For these networks, there is no distinction between the available wavelengths, i.e., the wavelength continuity constraint need not be satisfied along the lightpaths and the number of wavelengths on each link merely specifies a capacity constraint on the total number of lightpaths that can cross that link. Hence, these networks are mathematically no different than a circuit-switched network. The optimal routing-wavelength assignment problem for such networks reduces to finding a route for each lightpath (without assigning a specific wavelength) such that the resulting flows satisfy the capacity constraints. (For optical networks, flow is actually measured in terms of the number of lightpaths, i.e., flow on a link corresponds to the number of lightpaths that cross that link and flow of a path corresponds to the number of lightpaths that use that path.)

This problem can be formulated as follows: Suppose we have a connected undirected graph $G=(V, E)$, where $V$ denotes the set of nodes and $E$ denotes the set of edges. Each edge represents a pair of unidirectional fiber links in opposite directions. We are given set of origin-destination (OD) pairs, where an OD pair is an ordered pair $w=(i, j)$ of distinct nodes $i$ and $j$. Let $r_{w}$ denote the input traffic of OD pair $w$, which is a nonnegative integer representing the given number of lightpath requests of node $i$ destined for node $j$. We assume that lightpath requests are unidirectional, i.e., a lightpath request from node $i$ to node $j$ does not imply a lightpath request from node $j$ to node $i$. Denote

$$
\begin{aligned}
& W=\text { Set of all OD pairs, } \\
& P_{w}=\text { Set of paths that OD pair } w \text { may use, } \\
& C=\text { Set of wavelengths/colors available on each link. }
\end{aligned}
$$

The problem can be formulated in terms of a collection of path flows $\left\{x_{p} \mid w \in W, p \in P_{w}\right\}$, where $x_{p}$ represents the flow of path $p \in P_{w}$ for some $w \in W$ and takes a nonnegative integer value. The total flow on $\operatorname{link} l \in L, f_{l}$, 
can be expressed in terms of the path flows traversing link $l$ as

$$
f_{l}=\sum_{\{p \mid l \in p\}} x_{p}
$$

where we write $l \in p$ if link $l$ belongs to path $p$. Then, the problem takes the following form:

$$
\begin{aligned}
\text { minimize } & \sum_{l \in L} D_{l}\left(f_{l}\right) \\
\text { subject to } & \sum_{\{p \mid l \in p\}} x_{p} \leq|C|, \quad \text { for all } l \in L, \\
& \sum_{p \in P_{w}} x_{p}=r_{w}, \quad \text { for all } w \in W, \\
& x_{p}: \text { nonnegative integer, } \quad \text { for all } p \in P_{w}, w \in W,
\end{aligned}
$$

where $|C|$ denotes the cardinality of set $C$, i.e., the number of available wavelengths. The first constraint represents the capacity constraint on each link given by the number of available wavelengths, whereas the second constraint represents the requirement that the demand of each OD pair be satisfied by the resulting path flows.

In the above formulation, the overall cost function is given by the sum of link cost functions and each of the link cost functions depends on the amount of flow on the link. For this problem, we choose the link cost functions to have the piecewise linear form illustrated in Fig. 2. This cost function has two key features that impact significantly on the nature of the optimal solution:

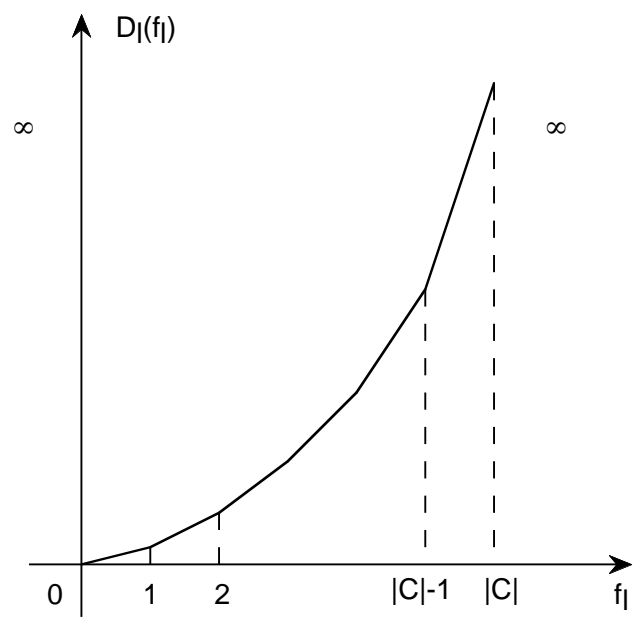

Figure 2. Piecewise linear cost function for link $l$. The function is convex and the break points occur at the integers $0,1, \ldots,|C|$, where $|C|$ denotes the number of available wavelengths. The cost for flow larger than $|C|$ is $\infty$.

(a) The cost function of every link is convex, monotonically increasing, and piecewise linear. Thus, the marginal cost for routing a new lightpath over a given link is larger than the marginal cost for routing the preceding lightpaths on the same link.

(b) The breakpoints of each piecewise linear link cost function occur at the integer points $0,1, \ldots,|C|$ (see Fig. 2). The cost for flow larger than $|C|$ is $\infty$, thereby imposing a link capacity constraint.

Because of feature (a), the resulting optimal solution of the associated linear program, favors choosing paths with underutilized links, and tends to leave room for future lightpaths. Because of feature (b), the resulting optimal 
solution tends to be integer, as we will explain shortly, thereby obviating the need for time-consuming integer programming techniques.

In optical networks with no wavelength conversion, the above path flow formulation needs to be modified because of the wavelength continuity constraint that needs to be satisfied along the lightpaths. For such networks, the path flows need to be distinguished by wavelength/color as well (i.e., wavelength assignment problem). Therefore, we formulate the routing-wavelength assignment problem for optical networks with no wavelength converters in terms of a path-wavelength vector

$$
\left\{x_{p}^{c} \mid p \in P_{w}, w \in W, c \in C\right\}
$$

The variable $x_{p}^{c}$ takes a value of 0 or 1 , and its meaning is

$$
x_{p}^{c}= \begin{cases}1, & \text { if wavelength } c \text { is used by path } p, \\ 0, & \text { otherwise }\end{cases}
$$

The total flow on link $l \in L, f_{l}$, can be expressed in terms of the $x_{p}^{c}$ as

$$
f_{l}=\sum_{\{p \mid l \in p\}} \sum_{c \in C} x_{p}^{c}
$$

Then, the problem formulation is given by

$$
\begin{aligned}
\text { minimize } & \sum_{l \in L} D_{l}\left(f_{l}\right) \\
\text { subject to } & \sum_{\{p \mid l \in p\}} x_{p}^{c} \leq 1, \quad \text { for all } l \in L, c \in C, \\
& \sum_{c \in C} \sum_{p \in P_{w}} x_{p}^{c}=r_{w}, \quad \text { for all } w \in W, \\
& x_{p}^{c}: 0 \text { or } 1, \quad \text { for all } p \in P_{w}, w \in W, c \in C,
\end{aligned}
$$

where $D_{l}$ is a piecewise linear, monotonically increasing, convex function, with break points at $0,1, \ldots,|C|$, as shown in Fig. 2. Here, the first constraint represents the capacity constraint that each wavelength on each link can be used at most once, whereas the second constraint represents the demand constraint of OD pairs.

Finally, let us consider networks with sparse wavelength conversion, i.e., only a fraction of the network nodes are equipped with wavelength converters. For these networks, we have the additional freedom of switching wavelength channels along the lightpaths at the nodes with converters. Therefore, in the corresponding problem formulation, we introduce more granularity in the optimization variables in order to distinguish nodes that have converters. More precisely, the problem is formulated in terms of a path-link-wavelength vector

$$
\left\{x_{p, l}^{c} \mid p \in P_{w}, w \in W, l \in L, c \in C\right\} .
$$

The variable $x_{p, l}^{c}$ takes a value of 0 or 1 , and its meaning is

$$
x_{p, l}^{c}=\left\{\begin{array}{l}
1, \text { if wavelength } c \text { is used on link } l \text { by path } p \\
0, \text { otherwise }
\end{array}\right.
$$


The total flow on link $l \in L, f_{l}$, can be expressed in terms of the $x_{p, l}^{c}$ as

$$
f_{l}=\sum_{\{p \mid l \in p\}} \sum_{c \in C} x_{p, l}^{c}
$$

In what follows, we write $\left(l_{1}, l_{2}\right) \in p$ if links $l_{1}$ and $l_{2}$ are successive links of path $p$. Also $l_{p}(1)$ represents the first link along path $p$. The corresponding problem formulation for this case is

$$
\begin{aligned}
\text { minimize } & \sum_{l \in L} D_{l}\left(f_{l}\right) \\
\text { subject to } & \sum_{\{p \mid l \in p\}} x_{p, l}^{c} \leq 1, \quad \text { for all } l \in L, c \in C, \\
& \sum_{c \in C} \sum_{p \in P_{w}} x_{p, l_{p}(1)}^{c}=r_{w}, \quad \text { for all } w \in W, \\
& x_{p, l}^{c}: 0 \text { or } 1, \quad \text { for all } p \in P_{w}, w \in W, l \in L, c \in C,
\end{aligned}
$$

where $D_{l}$ is a piecewise linear, monotonically increasing, convex function, with break points at $0,1, \ldots,|C|$, as shown in Fig. 2. Again the first and the second constraints represent the capacity and the demand constraints, respectively. We also impose the following conservation of flow constraints at the intermediate nodes. These constraints take different forms depending on whether or not there is a wavelength converter at that node.

At nodes with wavelength conversion, we have

$$
\sum_{c \in C} x_{p, l_{1}}^{c}=\sum_{c \in C} x_{p, l_{2}}^{c}, \quad \text { for all } p \in P_{w}, w \in W, \text { and all successive links }\left(l_{1}, l_{2}\right) \in p \text {. }
$$

At nodes without wavelength conversion, we have

$$
x_{p, l_{1}}^{c}=x_{p, l_{2}}^{c}, \quad \text { for all } p \in P_{w}, w \in W, c \in C \text {, and all successive links }\left(l_{1}, l_{2}\right) \in p \text {. }
$$

Note that the problem formulations given above also include the constraint that each variable $\left(x_{p}, x_{p}^{c}\right.$, or $\left.x_{p, l}^{c}\right)$ must be integer. This constraint is due to the fact that in practice, it is not allowed to bifurcate the traffic of an OD pair between alternative paths or wavelength channels. We will also consider the versions of these problems, where the integrality constraints are relaxed to

$$
\begin{aligned}
& 0 \leq x_{p}, \quad \text { for all } p \in P_{w}, w \in W, \\
& 0 \leq x_{p}^{c} \leq 1, \quad \text { for all } p \in P_{w}, w \in W, c \in C, \\
& 0 \leq x_{p, l}^{c} \leq 1, \quad \text { for all } p \in P_{w}, w \in W, c \in C,, l \in L,
\end{aligned}
$$

respectively, which we refer to as the corresponding relaxed problems. Because the cost function is piecewise linear and the constraints are linear, the relaxed problems can be converted to linear programs (LP), which can be solved by the simplex method or other polynomial complexity methods. Optimal solutions of the relaxed problems may involve some of the variables $\left(x_{p}, x_{p}^{c}\right.$, or $\left.x_{p, l}^{c}\right)$ being noninteger. We will argue, however, that at least in some practically important special cases, there exist optimal solutions of the relaxed problems that are integer, and therefore are also optimal solutions of the corresponding integer-constrained problems. 
We finally note that the formulations given above can be used both for the static traffic, where we need to process a collection of requests simultaneously, and for the dynamic traffic, where the lightpath requests arrive randomly in time. In the dynamic setting, some lightpaths could be currently established, and one or more new lightpath requests could be generated, whose routing-wavelength assignment schemes are subject to optimization. In practice, we would like to do this without disturbing the assignment for the existing lightpaths. This can be done by solving the problem that corresponds to the new lightpath requests, without changing the assignment for the existing lightpaths.

\subsection{Addressing Infeasibility Using Exact Penalty Functions}

Networks with sparse wavelength conversion capability is interesting, since practical considerations prohibit using wavelength converters at all the routing nodes. We now address the question of infeasibility in the problem formulation (F3) (sparse wavelength conversion), due to limited wavelength conversion capability in the network. Generally, it may not be possible to support the given set of lightpath requests $\left\{r_{w} \mid w \in W\right\}$, and problem (F3) turns out to be infeasible, i.e., there exists no path-wavelength assignment scheme for which the given constraints are satisfied simultaneously. Infeasibility generally arises because of inadequacy in the number of wavelength converters in the network, which requires that wavelength continuity constraint be satisfied along more lightpaths with no converters. With this in mind, we introduce another formulation, with fewer constraints, using the idea of penalty functions. Basically, we eliminate the conservation of flow constraints at nodes with no wavelength converters and add to the cost function a penalty term that prescribes a high cost to assignments which violate these constraints. Associated with the penalty term in the cost function is a penalty parameter $\gamma$ that determines the degree of penalty and as a result, the extent to which this alternative penalized problem approximates the original. As $\gamma$ increases, the approximation becomes more accurate. Using $f_{l}=\sum_{\{p \mid l \in p\}} \sum_{c \in C} x_{p, l}^{c}$, which represents the total flow on link $l$, in the cost function $\sum_{l \in L} D_{l}\left(f_{l}\right)$, we arrive at the following penalized cost function:

$$
\sum_{l \in L} D_{l}\left(\sum_{\{p \mid l \in p\}} \sum_{c \in C} x_{p, l}^{c}\right)+\gamma \sum_{c \in C} \sum_{w \in W} \sum_{p \in P_{w}} \underbrace{\sum_{\left(l_{1}, l_{2}\right) \in p}\left|x_{p, l_{1}}^{c}-x_{p, l_{2}}^{c}\right|}_{\text {at nodes without converters }}
$$

Classical optimization results, discussed in linear and nonlinear programming texts (see for example [Ber99]), state that the relaxed version of problem (F3) admits an exact penalty, meaning that when the relaxed problem is feasible and the scalar $\gamma>0$ is large enough, the corresponding penalized problem has the same solutions as the original relaxed problem. Hence if our problem is feasible, we can find the routing-wavelength assignment using the alternative formulation with the penalty function, provided that we choose the penalty parameter $\gamma$ sufficiently

large. Suppose now that we add conservation of flow constraints at nodes with no converters, pretending there are wavelength converters at these nodes. The optimal solution of the problem does not change, since it already satisfies these constraints. We thus arrive at the following penalized formulation: 


$$
\begin{aligned}
& \text { minimize } \sum_{l \in L} D_{l}\left(\sum_{\{p \mid l \in p\}} \sum_{c \in C} x_{p, l}^{c}\right)+\gamma \sum_{c \in C} \sum_{w \in W} \sum_{p \in P_{w}} \underbrace{\sum_{\left(l_{1}, l_{2}\right) \in p}\left|x_{p, l_{1}}^{c}-x_{p, l_{2}}^{c}\right|}_{\text {at nodes without converters }} \\
& \text { subject to } \quad \sum_{\{p \mid l \in p\}} x_{p, l}^{c} \leq 1, \quad \text { for all } l \in L, c \in C \\
& \sum_{c \in C} \sum_{p \in P_{w}} x_{p, l_{p}(1)}^{c}=r_{w}, \quad \text { for all } w \in W \\
& \sum_{c \in C} x_{p, l_{1}}^{c}=\sum_{c \in C} x_{p, l_{2}}^{c}, \quad \text { for all } p \in P_{w}, \text { and all successive links }\left(l_{1}, l_{2}\right) \in p
\end{aligned}
$$

and the constraints $x_{p, l}^{c} \in\{0,1\}$ or their relaxed versions $0 \leq x_{p, l}^{c} \leq 1$ for all $p \in P_{w}, w \in W, l \in L$, and $c \in C$.

The penalized problem has the advantage that it may have a feasible solution even when the original problem (F3) is infeasible. In particular, if the original problem (F3) is feasible and has some optimal solutions, the penalized formulation has the same optimal solutions (assuming $\gamma$ is large enough). If the original problem is infeasible, i.e., there is no possible routing-wavelength assignment to satisfy the requests for a network with a given limited number of converters, the penalized problem may still have an optimal solution that involves wavelength conversions at some of the nodes where there are actually no converters. This solution is helpful during a network design process, because it indicates the best placement of extra converters in order to satisfy the given lightpath request set.

The exact penalty formulation can also be used within several other network design contexts. In particular, it can be used for reconfiguration purposes, in order to adapt to changes in traffic demands or network topology. Given a small change in the current operating conditions of the network, we would ideally like to maintain the routing-wavelength assignment for the existing lightpaths as much as possible. This can be achieved by introducing a penalty in the cost function that penalizes changes from the current assignment scheme. Then the algorithm tries to find the best assignment suitable for the new conditions in the network without deviating much from the previous assignment.

In particular, consider the dynamic setting where some lightpaths are currently established and operating, and one or more new lightpath requests are generated, and their routing and wavelength assignment must be considered. One possibility then is to place these new requests along whatever free paths and wavelengths can be found without rerouting any of the existing lightpaths. This can be attempted by either using a heuristic or by solving the problem that corresponds to just the new lightpath requests, with the bandwidth allocated to the current lightpaths undisturbed. On the other hand, this problem may turn out to be infeasible, and it may not be possible to satisfy the new lightpath requests without rerouting the existing lightpaths. In this case one may solve an RWA problem that involves all lightpaths, old and new, but with an exact penalty term that penalizes the rerouting of the existing lightpaths. This term has the form

$$
\gamma \sum_{\left\{w \in W, p \in P_{w}, l \in L, c \in C \mid \bar{x}_{p, l}^{c}=1\right\}}\left|x_{p, l}^{c}-\bar{x}_{p, l}^{c}\right|,
$$

where $\left\{\bar{x}_{p, l}^{c}\right\}$ are the current set of variables that correspond to the existing lightpaths and $\gamma$ is the penalty parameter. If $\gamma$ is large enough, it can be shown that the solution of the corresponding optimization problem will not reroute any of the existing lightpaths, if this is at all possible. 
The exact penalty function approach can also be used to determine the amount of resources/bandwidth required on each link in order to support a given traffic pattern. For this purpose, we first assume an initial reasonably small value $|C|$ for the number of available wavelengths on each link. If the number of lightpath requests is excessive relative to the number $|C|$ of available wavelengths, it will be impossible to satisfy all of these requests, even with wavelength conversion capability at each node. Therefore, we introduce a sufficient number of additional wavelengths, but with an associated incremental cost (slope of the piecewise linear cost function) that is so high that these wavelengths are not used in an optimal solution if it is at all possible to satisfy the given lightpath requests with the existing number $|C|$ of wavelengths. When, however, the number $|C|$ is inadequate, the optimal solution of the penalized problem will provide an indication of the minimal number of additional wavelengths needed to satisfy the given lightpath requests.

\subsection{Obtaining Integer Solutions}

The use of piecewise linear objective functions $D_{l}$ with integer break points has some important consequences. First, the corresponding relaxed linear programming (LP) model, where the integer constraints are replaced by the relaxed constraints [cf. Eq. (2.1)], can be solved by efficient commercial or special purpose simplex methods with fast running times. Second, even if we relax the integer constraints, it appears that an integer optimal solution can still be obtained in most cases of interest. We have verified this by analysis, and computational experiment using a simplex code (CPLEX). In all of the experiments we performed with a variety of network topologies, where the input traffic of OD pairs were randomly selected, the corresponding relaxed LP problem, in any of the given formulations, yielded integer solutions. We have also proved the integrality of optimal solutions analytically for the case of general ring networks with multiple origins and destinations under some assumptions. We provide in the next section a counterexample where the relaxed version of problem (F1) with full wavelength conversion does not

have an integer optimal solution. This extreme case arises due to symmetry in the location of OD pairs and heavy traffic requests.

Thus, our research indicates that for most of the cases, the relaxed problem, in any of the given formulations, has an integer optimal solution (assuming it has a feasible solution). The reason is that, because of the structure of the piecewise linear cost function, extreme points of the relaxed constraint polyhedron appear to be integer in the majority of cases. Intuitively, the extreme points of the constraint set tend to correspond to the corner points of the piecewise linear objective function, which take integer values. Note that, as can be seen from simple examples, other types of link cost functions (e.g., $D_{l}$ : linear, or smooth and convex), such as those commonly used in optimal data network routing (see for example [BeG92]), typically result in fractional optimal solutions for the relaxed versions of these formulations.

We finally mention that even in the cases where the solution to the relaxed problem may be fractional, the number of fractional variables in the solution typically turn out to be insignificant relative to the number of integer variables. As a result, it may be possible to round the fractional portion of the solution to integer with the use of simple heuristics. Indeed, in Section 4, we provide a simple rounding method that takes into account the structure of the cost function, and starting from a fractional optimal solution, produces an integer solution with no or little loss of optimality. 


\section{INTEGER SOLUTION FOR SOME NETWORK TOPOLOGIES}

In this section, we focus our attention to specific network topologies such as line and ring networks. We consider networks with full wavelength conversion or no wavelength conversion capabilities [i.e., we analyze the problem formulations (F1) and (F2) given in the preceding section]. We show that under certain assumptions, the relaxed versions of problems (F1) and (F2) have integer optimal solutions, which are also optimal for the integer-constrained problems. Recall that we represent a network with a connected undirected graph $G=(V, E)$ ( $V$ denoting the set of nodes and $E$ denoting the set of edges) with a given set of OD pairs, where each OD pair is an ordered pair $(i, j)$ of distinct nodes $i$ and $j$. We assume that each OD pair has one unit of input traffic.

\section{Line Network}

We first consider the case where $G$ is a line, i.e., the nodes $1, \ldots, n$ of $G$ are linearly arranged so that each node $i$ is directly connected to $i-1$ and $i+1$, except if $i=1$ or $i=n$, in which case $i$ is directly connected to node 2 or node $n-1$, respectively. In the line network, there is a single available path for each OD pair. Therefore, given a set of OD pairs, the set of paths are determined and we are interested in the wavelength assignment problem only. Recall that a wavelength assignment for a given set of paths assigns wavelengths to each link of each path such that no link gets the same wavelength for two different paths passing through it. We say that a wavelength assignment has no wavelength conversion if every path is assigned the same wavelength on all of its links.

An interesting result, shown in the context of graph coloring problems, is that if the number of paths crossing every link is less than or equal to the number of available wavelengths [a necessary condition for feasibility of both problems (F1) and (F2)], then there is a wavelength assignment with no wavelength conversion [Tuc75]. In other words, the problem is feasible with wavelength conversion if and only if it is feasible without wavelength conversion. Since this result is important for our purposes, we prove it constructively with an efficient algorithm.

Proposition 1: Consider a line network with multiple OD pairs. Suppose that the number of paths crossing any link is less than or equal to the number of available wavelengths. Then there exists a wavelength assignment with no wavelength conversion.

Proof: Consider first the rightward directed paths determined by the given set of OD pairs and assume that these paths are ordered from left to right, based on the location of their origins. We assign wavelengths to paths consecutively in the following way: We start with path 1 and assign the first available wavelength to path 1 on all of its links. For path $k$, we search for an available wavelength on its first link. In view of the assumption that the number of paths crossing any link is less than or equal to the number of wavelengths, it is possible to find an available wavelength $\hat{c}$ on this link.

We claim that $\hat{c}$ is also available on all the subsequent links of path $k$ and therefore can be assigned to path $k$ on all these links. To see this, suppose that $\hat{c}$ is not available on one of the subsequent links. This means that wavelength $\hat{c}$ is assigned to path $i$ on this link, for some $i<k$, implying that $\hat{c}$ is also assigned to path $i$ on the first link of path $k$, which is a contradiction. A similar argument works for the leftward directed paths. Hence, there exists a wavelength assignment with no wavelength conversion. Q.E.D. 


\section{Ring Network with Full Wavelength Conversion}

Consider next the case where $G$ is a ring and there is full wavelength conversion capability at every node [problem (F1) of Section 2]. Any feasible solution of the relaxed version of problem (F1) specifies a set of paths together with the path flows that carry the input traffic of the OD pairs (also referred to as a routing). Any integer feasible solution corresponds to a routing in which all origins send their input traffic completely along a single path. Similarly, a fractional feasible solution (a feasible solution in which some of the variables are noninteger) corresponds to a routing in which some of the origins divide their traffic between alternative paths.

We consider a ring network with multiple OD pairs in which the origins and destinations are located such that the ring can be separated in two pieces by removing two links with one of the obtained pieces containing all the origins and the other containing all the destinations. We say that in such a network, the origins and the destination can be separated (see Fig. 3). In the following, we prove that for a ring network where the origins and the destinations can be separated, the relaxed version of problem (F1) has an integer optimal solution.

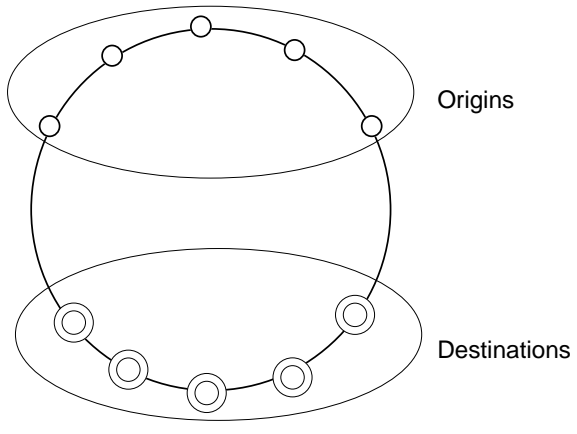

Figure 3. A ring network with multiple OD pairs, where origins and destinations can be separated.

We first show the following proposition related to optimal fractional solutions of the relaxed version of problem (F1). We say that $n O D$ pairs of the ring network interleave if it is not possible to separate the ring into two pieces by removing two links so that any two of the OD pairs are entirely contained in one of the pieces (see Fig. 4).

Proposition 2: Consider a ring network with multiple OD pairs such that the origins and the destinations can be separated. In any optimal fractional solution of the relaxed version of problem (F1) for this network, all OD pairs that divide their traffic between alternative paths interleave.

Proof: Suppose to arrive at a contradiction that two of the OD pairs, say OD pair 1 and 2, that split their traffic between alternative paths do not interleave, i.e., the ring can be separated into two pieces by removing two links such that each of the pieces obtained contains exactly one of these OD pairs [see Fig. 4(b)]. We consider another feasible solution obtained from the fractional optimal solution in the following way: we increase the traffic of OD pair 1 in the counterclockwise direction by a small amount $\delta$ while decreasing the traffic in the clockwise direction by the same amount. Similarly, we decrease the traffic of OD pair 2 in the counterclockwise direction by $\delta$, while increasing the traffic in the clockwise direction by $\delta$. (This can be done since the traffic of both OD pairs is nonzero in each direction in the routing specified by the fractional optimal solution.) The link flows corresponding to this feasible solution are the same as those corresponding to the fractional optimal solution on all the links, except on those along the clockwise path from origin 1 to origin 2 and from destination 2 to destination 
1, and the counterclockwise path from origin 2 to origin 1 and destination 1 to destination 2 , on which the flows are reduced. Since cost is an additive, monotonically increasing function of link flows, the feasible solution thus obtained has smaller cost value, contradicting the optimality of the starting fractional solution. Hence, in any optimal fractional solution of the relaxed version of problem (F1), all OD pairs that divide their traffic must be interleaving. Q.E.D.

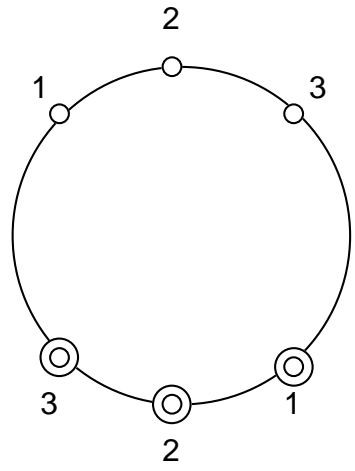

(a)

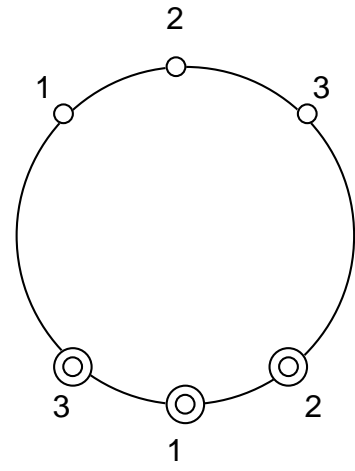

(b)

Figure 4. A ring network with three OD pairs. The circles represent the origins, whereas the double circles represent the destinations. In the ring network given in (a), the three OD pairs shown interleave, i.e., it is not possible to separate the ring into two pieces by removing two links so that any two of the OD pairs are entirely contained in one of the pieces. In the ring network given in (b), the three OD pairs shown do not interleave, i.e., the ring can be divided in two pieces by removing the link between the origins of OD pairs 1 and 2 , and the link between the destinations of OD pairs 1 and 2, such that each of the pieces obtained contains exactly one of these OD pairs.

Next, we consider solving the integer-relaxed version of problem (F1) by means of some polynomial complexity LP method. The resulting optimal solution of the relaxed problem may involve some of the variables being fractional. In the next proposition, we show that there exists an algorithm which, starting from a fractional optimal solution, produces an integer optimal solution.

Proposition 3: Consider a ring network with multiple OD pairs such that the origins and the destinations can be separated. If the relaxed version of problem (F1) is feasible, then it has an integer optimal solution [which is also optimal for the integer-constrained problem (F1)].

Proof: We prove this proposition by providing an algorithm that takes an optimal fractional solution of the problem that involves some OD pairs that divide their traffic between alternative paths and at each iteration produces another optimal solution with fewer OD pairs that divide their traffic.

For this purpose, we first introduce some notation. Suppose in the beginning of an iteration, we have a fractional optimal solution of the relaxed version of problem (F1) that involves $n$ OD pairs, denoted $1, \ldots, n$, that split their input traffic between two alternative paths, in addition to some other OD pairs that send all their traffic 
along a single path. For the $i^{\text {th }}$ OD pair that splits its traffic, denote the flow along the counterclockwise path by $x_{i}$, where $0<x_{i}<1$. We consider the general case where no two of the given OD pairs have the same origin and the same destination. [Otherwise, we can represent the common origin (or the common destination) by two origins (or two destinations) with a zero cost link in between.] Without loss of generality, assume that the origins of the OD pairs are arranged consecutively in the clockwise direction. Since by Proposition 2, all OD pairs that divide their traffic between alternative paths must interleave, it can be seen that the corresponding destinations must also be arranged consecutively from 1 to $n$ in the clockwise direction, as shown in Fig. 5, which also illustrates the link flows corresponding to a fractional optimal solution that involves three OD pairs that divide their traffic.

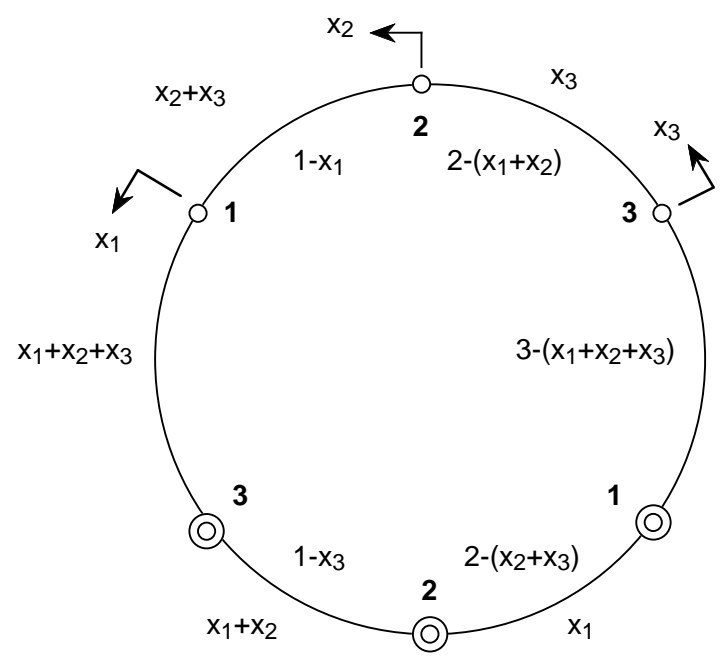

Figure 5. A ring network with three OD pairs, which interleave. The circles represent the origins, whereas the double circles represent the destinations. We consider a fractional optimal routing to the relaxed problem, in which the three OD pairs divide their traffic between the alternative paths. For this example, the flow along the counterclockwise path of the the $i^{\text {th }}$ OD pair is given by $x_{i}$ with $0<x_{i}<1$. The corresponding link flows are also illustrated in the figure. The counterclockwise (clockwise) flows are shown outside (inside) the ring.

The flows of the links that belong to paths used by any of the $n$ OD pairs $1, \ldots, n$ are equal to an integer plus or minus the following $2 n$ quantities:

$$
\begin{aligned}
& x_{1},\left(x_{1}+x_{2}\right), \ldots,\left(x_{1}+x_{2}+\cdots+x_{n-1}\right),\left(x_{1}+x_{2}+\cdots+x_{n-1}+x_{n}\right), \\
& \left(x_{2}+x_{3}+\cdots+x_{n-1}+x_{n}\right),\left(x_{3}+\cdots+x_{n-1}+x_{n}\right), \ldots, x_{n}, 0 .
\end{aligned}
$$

Let us represent the $2 n-1$ nonzero quantities in (3.1) compactly as

$$
a_{j}^{\prime} x, \quad j=1, \ldots, 2 n-1,
$$

where $x$ is the $n$-dimensional vector given by $x=\left(x_{1}, \ldots, x_{n}\right)$ and $a_{j}, j=1, \ldots, 2 n-1$, is an $n$-dimensional vector whose components are 0 or 1 . Let the index set $J(x)$ be given by

$$
J(x)=\left\{j \in\{1, \ldots, 2 n-1\} \mid a_{j}^{\prime} x=\text { integer }\right\} .
$$

One iteration of the algorithm consists of the following steps:

1) Find a nonzero vector $\Delta x$ that satisfies

$$
a_{j}^{\prime} \Delta x=0, \quad \text { for all } j \in J(x) .
$$


To see why such a nonzero vector exists note that, since $x_{1}, \ldots, x_{n}$ are noninteger, two successive quantities in collection (3.1) cannot be integer. Hence, this collection contains at most $(n-1)$ nonzero quantities that are integer. This implies that the set $J(x)$ contains at most $(n-1)$ indices, and therefore, the system of equations given in (3.2) has a nonzero solution $\Delta x$. Since $\Delta x$ satisfies Eq. (3.2), it follows that $J(x+\alpha \Delta x)=J(x)$ for some sufficiently small scalar $\alpha$, and the corresponding cost change, denoted by $\Delta$ cost, can be expressed as

$$
\Delta \operatorname{cost}=\alpha \sum_{j \notin J(x)} b_{j}^{k}\left(a_{j}^{\prime} \Delta x\right) \geq 0
$$

where $b_{j}^{k}$ is the slope of linear segment $k$ in the piecewise linear cost function for link $j$. The inequality follows from the assumption that the fractional optimal solution corresponding to $x$ is optimal. Since $-\Delta x$ also satisfies Eq. (3.2), the corresponding cost change is given by $-\Delta$ cost, and the preceding inequality holds with $\Delta x$ replaced by $-\Delta x$, implying that the cost change by moving along direction $\Delta x$ is zero. Hence, any $\Delta x$ satisfying (3.2) is a cost preserving direction at the optimal solution $x$, along which we can change $x$ without increasing the cost.

2) Let $\alpha$ be the smallest scalar such that, either any one of the components of the vector $x+\alpha \Delta x$ becomes integer or one of the link flows $a_{j}^{\prime}(x+\alpha \Delta x)$ for some $j \notin J(x)$ becomes integer. Replace $x$ by $x+\alpha \Delta x$ and $J(x)$ by $J(x+\alpha \Delta x)$. If all components of the resulting vector $x$ are integer, stop. Else, if at least one of the components of the vector $x$ is integer, go to the next iteration. [This corresponds to having a new optimal solution for the relaxed version of problem (F1), which has fewer fractional components.]

3) Else go back to step 1. [This corresponds to having a new optimal solution, which yields fewer fractional link flows.]

Since there is a finite number of link flows that are noninteger, step 3 is repeated finite number of times before we get an optimal solution which involves fewer OD pairs that divide their traffic between alternative paths and go to the next iteration. Since at the end of each iteration, we have an optimal solution with fewer fractional components, this algorithm yields an integer optimal solution for this network in finite number of iterations. Note also that in the above procedure, since we keep the integer link flows constant and modify the fractional link flows, no additional wavelength channels are required to support the modified link flows. The essence of the algorithm lies in the fact that because of the special topology of this network, there exist nonzero directions, along which we can change a fractional optimal solution of the relaxed version of problem (F1) and obtain another optimal solution that has fewer fractional components. Q.E.D.

However, for a general ring network with arbitrarily located OD pairs, it is not always true that if the integerrelaxed version of problem (F1) is feasible, then the problem has an integer optimal solution, as shown in the following example.

\section{Example 1:}

Consider the ring network of Figure 6. We have three OD pairs and one wavelength channel available in each direction of the links. Each OD pair has one unit of input traffic. We claim that for this network, problem (F1) is infeasible, 
i.e., it is not possible to send the input traffic of each OD pair along a single path. To see this, assume without loss of generality that origin 1 sends one unit of traffic along the clockwise path. Since one of the links along the clockwise path of OD pair 2 does not have any available wavelength channels, origin 2 sends its traffic along the counterclockwise path, and it is not possible to send the input traffic of OD pair 3 along any of its two paths. On the other hand, if each origin divides its input traffic equally between the alternative paths, the flow of each link becomes either 1 or $1 / 2$, showing that the integer-relaxed version of problem $(\mathrm{F} 1)$ is feasible.

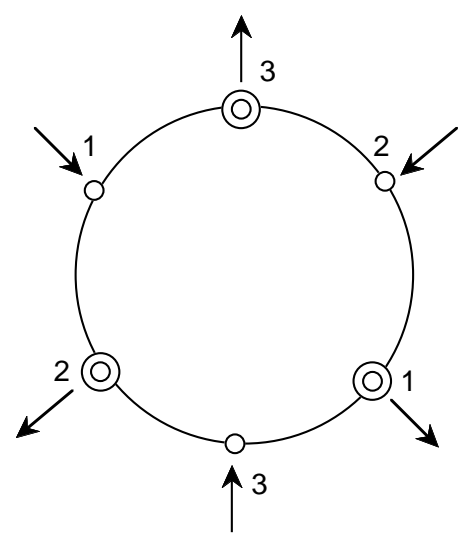

Figure 6. A ring network for which the relaxed version of problem (F1) is feasible, but does not have an integer optimal solution. The circles represent the origins, whereas the double circles represent the destinations.

\section{Ring Network with No Wavelength Conversion}

Next we consider the case where $G$ is a ring and there is no wavelength conversion capability at any of the nodes [problem (F2) of Section 2]. We have the following result for ring networks with no wavelength conversion capability at any of the nodes.

Proposition 4: Consider a ring network with multiple OD pairs such that the origins and the destinations can be separated. If the relaxed version of problem (F1) with full wavelength conversion capability is feasible, then the relaxed version of problem (F2) has an integer optimal solution.

Proof: By Proposition 3, it follows that the relaxed version of problem (F1) has an integer optimal solution. This solution specifies a routing, in which all origins send their input traffic completely along a single path. For this topology, the paths in the clockwise and counterclockwise directions can be ordered, and the wavelength allocation algorithm for the line network (cf. proof of Proposition 1) can be used to find a wavelength assignment with no wavelength conversion. The resulting routing-wavelength assignment gives an integer optimal solution of the relaxed version of problem (F2). Q.E.D.

\section{ARBITRARY NETWORK TOPOLOGIES}

We next address the question of finding optimal or near-optimal solutions of problems (F1) and (F2) for arbitrary network topologies. As mentioned in Section 2.2, our computational experience suggests that for most network topologies, solving the relaxed versions of any of these formulations using the efficient simplex method yields integer optimal solutions. However, there may be some network topologies for which the relaxed problems do 
not have integer optimal solutions (cf. Example 1). In this section, we provide an algorithm that uses the algorithm given in the proof of Proposition 3 of the previous section and yields an integer optimal (or slightly suboptimal) solution for problem (F1) (full wavelength conversion case) for arbitrary network topologies. This algorithm may also be used to construct effective methods that compute optimal or near-optimal solutions for the no wavelength conversion case, as we discuss at the end of the present section.

Suppose we have an arbitrary network with a given set of OD pairs and a given number of wavelength channels on the links. We assume that we have full wavelength conversion capability at all of the nodes of the network. We use the following algorithm to find an optimal or near-optimal solution of problem (F1). We assume without loss of generality that each OD pair has one unit of input traffic. (An OD pair which has several units of input traffic can be represented in terms of several OD pairs which have the same origin and the same destination, and which has one unit of input traffic each.) We also assume that there are some extra wavelength channels on the links, which can be used in the algorithm. This entails no loss of generality, since these wavelengths can be introduced with an associated incremental cost (slope of piecewise linear link cost function) that is so high that these wavelengths are not used in an optimal solution of problem (F1). Recall that a feasible solution of problem (F1) has the form $x=\left\{x_{p} \mid p \in P_{w}, w \in W\right\}$, where $W$ denotes the set of all OD pairs, and $P_{w}$ denotes the set of paths that some OD pair $w \in W$ may use.

At the start of iteration $k$, we have the subset $S_{k}$ of path flow variables (the $x_{p}$ ), which are subject to optimization, and the complementary set of the variables not in $S_{k}$, which have been permanently fixed at 0 or 1 . Initially $S_{0}$ is the set of all path flow variables. The $k^{t h}$ iteration of the algorithm consists of the following steps:

1) Solve the relaxed version of problem (F1) for the path flow variables in $S_{k}$, while the remaining path flow variables are fixed at 0 or 1 . Let $x=\left\{x_{p} \mid p \in P_{w}, w \in W\right\}$ denote the corresponding optimal solution. If all the $x_{p}$ are integer, stop. Else go to step 2.

2) At this step, we use an iterative procedure based on the algorithm given in the proof of Proposition 3 to round the fractional components of the vector $x$ without changing its cost. In particular, we search for cost preserving directions, along which we can change $x$ and obtain other optimal solutions that involve fewer fractional components (cf. proof of Proposition 3). This procedure consists of the following steps:

2a) Let $\bar{W}$ be the set of OD pairs that have some fractional path flow variables in the current solution $x$, and for each $w \in \bar{W}$, denote the set of paths that carry nonzero flows by $\bar{P}_{w}$, i.e., $x_{p} \in(0,1)$ for all $p \in \bar{P}_{w}$ and all $w \in \bar{W}$. Let $\bar{x}=\left\{x_{p} \mid p \in \bar{P}_{w}, w \in \bar{W}\right\}$ be a vector with $m$ components, consisting of the fractional components of the current solution $x$. Denote $\bar{L}$ to be the set of network links that belong to paths $p \in \bar{P}_{w}$ for all $w \in \bar{W}$. The flows of the links $j \in \bar{L}$ can be represented by an integer plus or minus the following quantities,

$$
a_{j}^{\prime} \bar{x}, \quad j \in \bar{L},
$$

where $a_{j}, j \in \bar{L}$, is an $m$-dimensional vector whose components are 0 or 1 . Let the index set $J(\bar{x})$ be given by

$$
J(\bar{x})=\left\{j \in \bar{L} \mid a_{j}^{\prime} \bar{x}=\text { integer }\right\} .
$$


If $J(\bar{x})$ is empty, then let $d$ be any $m$-dimensional vector and go to step 2c. Else go to step 2b.

$2 \mathrm{~b})$ Search for a nonzero vector $d$ that satisfies the set of equations

$$
a_{j}^{\prime} d=0, \quad \forall j \in J(\bar{x})
$$

If there exists such a nonzero vector, go to step 2c. Else go to step 3. (A cost preserving direction cannot be found.)

2c) Let $\alpha$ be the smallest scalar such that, either any one of the components of the vector $\bar{x}+\alpha d$ becomes integer or one of the link flows $a_{j}^{\prime}(\bar{x}+\alpha d)$ for some $j \notin J(\bar{x})$ becomes integer. Replace $\bar{x}$ by $\bar{x}+\alpha d$ and $J(\bar{x})$ by $J(\bar{x}+\alpha d)$. Change the current solution $x$ by replacing the corresponding components with those of $\bar{x}$. If all components of the vector $\bar{x}$ are integer, stop. Else if at least one of the components of the vector $\bar{x}$ is integer, go back to step 2a. [This corresponds to having a new optimal solution for the relaxed version of problem (F1) solved in step (1), which involves fewer fractional components.] Else go to step $2 \mathrm{~d}$.

2d) Go back to step 2b. [This corresponds to having a new optimal solution, which yields fewer fractional link flows. Since there is a finite number of fractional link flows, this step will be repeated finitely many times before the number of fractional components of the current solution is reduced.]

3) Consider the set $\bar{W}$ of OD pairs that have some fractional path flow variables in the current solution $x$. Find some $\bar{w} \in \bar{W}$ such that when setting $x_{\bar{p}}$ to 1 , for some $\bar{p} \in P_{\bar{w}}$, and the $x_{p}$ to 0 , for all $p \in P_{\bar{w}}$ with $p \neq \bar{p}$, the change in the cost is the most favorable. Change the current solution $x$ by replacing $x_{\bar{p}}$ by 1 and $x_{p}$ by 0 for all $p \in P_{\bar{w}}$ with $p \neq \bar{p}$. Let $S_{k+1}$ be the set of noninteger components of the vector $x$ and go to the next iteration.

At the termination of this algorithm, the resulting solution $x$ specifies a routing, in which a single path is assigned to the input traffic of each OD pair. In essence, the algorithm solves the relaxed version of problem (F1) (step 1) and searches for a cost preserving direction to round the fractional components of the resulting optimal solution (step 2). Whenever a cost preserving direction cannot be found, it chooses one OD pair that has some fractional path flow variables and rounds all of its variables to 0 or 1 (step 3). Then it resolves the relaxed problem with all the current integer variables fixed with the aim of rectifying poor routing patterns that have been created by rounding. Note that the number of wavelengths needed on the links may increase in step 3. This is the reason why we had to assume that we have extra wavelengths on the links. The algorithm may be viewed as a generalization of the one given for a ring network in which the origins and the destinations can be separated. In fact, for such a network, the algorithm terminates either at step 1 or step 2 with an integer optimal solution of problem (F1).

Let us also note a more powerful but also a computationally more intensive variant of the algorithm, which combines steps 3 and 1 . Here, upon entering step 3, for each $\bar{w} \in \bar{W}$ and each $\bar{p} \in P_{\bar{w}}$, we set $x_{\bar{p}}=1$ and $x_{p}=0$ for all $p \in P_{\bar{w}}$ with $p \neq \bar{p}$, and we solve the corresponding relaxed version of problem (F1) for the remaining path flow variables in $S_{k}$. We find the OD pair $\bar{w}$ and path $\bar{p}$ that results in the most favorable cost change, and proceed from here as in Step 3. This version of the algorithm may be viewed as a special case of a rollout algorithm, which is a 
general method for enhancing the effectiveness of heuristics (see [Ber98], [Ber00]). For the remainder of this paper, we will focus on the first variant of the algorithm. We now illustrate this routing algorithm by various examples.

\section{Example 2.}

Consider the network of Example 1, where we have a ring network with three OD pairs having one unit of input traffic each (cf. Figure 6). We assume that there is one wavelength channel with small incremental cost and additional wavelength channels with very high incremental cost in each direction of each link. For the $i^{\text {th }}$ OD pair, denote the flow along the counterclockwise path by $x_{i}$.

Let $S_{0}=\left\{x_{1}, x_{2}, x_{3}\right\}$ and solve the relaxed version of problem (F1) for the path flow variables in $S_{0}$ for this network (step 1 of the algorithm). In terms of the variables in $S_{0}$, the flows on the links of this network are equal to

$$
\begin{gathered}
x_{1}, x_{2}, x_{3}, 1-x_{1}, 1-x_{2}, 1-x_{3}, \\
x_{1}+x_{2}, x_{1}+x_{3}, x_{2}+x_{3}, 2-\left(x_{1}+x_{2}\right), 2-\left(x_{1}+x_{3}\right), 2-\left(x_{2}+x_{3}\right) .
\end{gathered}
$$

Since there is only one wavelength channel with small incremental cost on the links and the vector $x=[1 / 2,1 / 2,1 / 2]$ is the only solution for which all the link flows are less than or equal to one, it follows that $x$ is an optimal solution of the relaxed problem. Next we search for a nonzero direction along which we can change $x$ without changing the cost (step 2). The link flows in the second line of the above collection are integer at the current solution $x$. Therefore, we search for a nonzero vector $d=\left[d_{1}, d_{2}, d_{3}\right]$ that satisfies

$$
d_{1}+d_{2}=0, \quad d_{1}+d_{3}=0, \quad d_{2}+d_{3}=0,
$$

(step $2 \mathrm{~b}$ ). Since there is no nonzero vector $d$ that satisfies these equations, we search for some OD pair $i$, where $i=1,2$, or 3 , such that rounding $x_{i}$ to 0 or 1 gives the most favorable cost change (step 3). Due to symmetry of the location of OD pairs and the symmetry of the link flows at the current solution, it can be seen that setting any one of the $x_{i}$ 's to 0 or 1 yields the same amount of cost change. Therefore, we choose to set $x_{1}$ to 1 and update $x$ to $x=[1,1 / 2,1 / 2]$. Let $S_{1}=\left\{x_{2}, x_{3}\right\}$ (the noninteger components of the resulting vector $x$ ) and resolve the relaxed problem for the path flow variables in $S_{1}$, while $x_{1}$ is fixed at 1 . In terms of the variables in $S_{1}$, the flows of the links that belong to paths used by OD pairs 2 and 3 are equal to an integer plus or minus the following quantities:

$$
\begin{aligned}
& x_{2}, x_{3}, \\
& x_{2}+x_{3} .
\end{aligned}
$$

It can be seen that $x=[1,1 / 2,1 / 2]$ is an optimal solution of this relaxed problem. (This can be verified analytically or computationally using a simple LP code.) Since at the current solution $x$, the only quantity that is integer in the above collection is $x_{2}+x_{3}$, we let $d=\left[d_{2}, d_{3}\right]$ be any vector that satisfies $d_{2}+d_{3}=0$. In particular, we choose $d=[1,-1]$ and change $\left[x_{2}, x_{3}\right]=[1 / 2,1 / 2]$ to $\left[x_{2}, x_{3}\right]+1 / 2\left[d_{2}, d_{3}\right]=[1,0]$. We update $x$ to $x=[1,1,0]$ by replacing the corresponding components. (This is another optimal solution of the relaxed problem, which is solved for the variables in $S_{1}$ with $x_{1}$ fixed at 1.) Since all components of $x$ are integer, we stop. The resulting integer solution is also an integer optimal solution of problem (F1).

\section{Example 3.}

Consider the ring network of Figure 7. We have three OD pairs that send their one unit of input traffic along a single path and three OD pairs $(1,2$, and 3$)$ with one unit of input traffic each, whose routing is subject to optimization. 

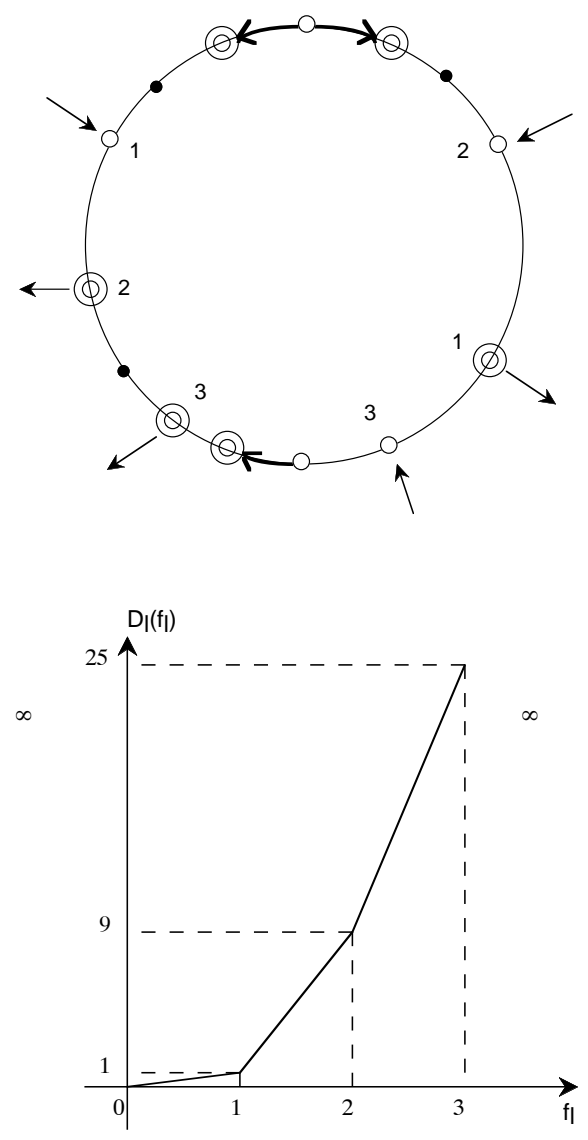

Figure 7. A ring network with three OD pairs that send their one unit of input traffic along a single path and three OD pairs (1,2, and 3$)$ with one unit of input traffic each, whose routing is subject to optimization. The circles represent the origins, whereas the double circles represent the destinations. Solid dots represent the nodes which do not transmit or receive any traffic.
Figure 8. Piecewise linear cost function for each link of the network of Figure 7 .

Each link cost function has the piecewise linear form given in Figure 8. For the $i^{\text {th }}$ OD pair, denote the flow along the counterclockwise path by $x_{i}$.

Let $S_{0}=\left\{x_{1}, x_{2}, x_{3}\right\}$ and solve the relaxed version of problem (F1) for the path flow variables in $S_{0}$ for this network. In terms of the variables in $S_{0}$, the flows on the links of this network are equal to an integer plus or minus the following quantities:

$$
\begin{gathered}
x_{1}, x_{2}, x_{3}, \\
x_{1}+x_{2}, x_{1}+x_{3}, x_{2}+x_{3}, \\
x_{1}+x_{2}+x_{3} .
\end{gathered}
$$

The vector $x=[1 / 2,1 / 2,1 / 2]$ is an optimal solution of this problem with optimal cost equal to 45 . (This can be verified analytically or computationally using a simple LP code.) The quantities in the second line of the above collection are integer at the current solution $x$. Therefore, we search for a nonzero vector $d=\left[d_{1}, d_{2}, d_{3}\right]$ that satisfies

$$
d_{1}+d_{2}=0, \quad d_{1}+d_{3}=0, \quad d_{2}+d_{3}=0
$$

Since there is no nonzero vector $d$ that satisfies these equations, we search for some OD pair $i$, where $i=1,2$ or 3 , such that rounding $x_{i}$ to 0 or 1 gives the most favorable cost change. There are six different ways of rounding one of the $x_{i}$ 's to 0 or 1 . The resulting vectors and the costs are given as follows:
$[0,1 / 2,1 / 2]$ with cost 48.5
$[1,1 / 2,1 / 2]$ with cost 55.5
$[1 / 2,0,1 / 2]$ with cost 54 ,
$[1 / 2,1,1 / 2]$ with cost 79 ,
$[1 / 2,1 / 2,0]$ with cost 50.5 ,
$[1 / 2,1 / 2,1]$ with cost 75.5 . 
Rounding $x_{1}$ to 0 gives the least amount of cost change, so we set $x_{1}$ equal to 0 , and update $x$ to $x=[0,1 / 2,1 / 2]$, which has a cost of 48.5. Let $S_{1}=\left\{x_{2}, x_{3}\right\}$ and resolve the relaxed problem for the path flow variables in $S_{1}$, while $x_{1}$ is fixed at 0 . It can be verified computationally that the vector $x=[0,1,0]$ is an optimal solution of this problem with optimal cost 45, which is also the integer optimal solution of problem (F1) for this network. Note that in this example, resolving the relaxed problem after rounding one of the variables reduces the cost of the current solution, thus rectifying the poor routing resulting from rounding. If we did not resolve the relaxed problem and instead tried to find a direction using step 2 of the algorithm, we could have obtained the vector $x=[0,0,1]$, which is an integer solution of problem (F1) with cost 51. The reason is that when the current solution is not an optimal solution of the relaxed problem, step 2 need not yield a cost preserving direction and we may end up with an integer solution which is far from optimal.

\section{Example 4.}

Consider the network of Figure 9, which is a ring that includes two subrings. Denote the subring at the top by $R_{1}$ and the subring at the bottom by $R_{2}$. Each OD pair has one unit of input traffic. We assume that there is one wavelength channel with small incremental cost and additional wavelength channels with very high incremental cost in each direction of each link. By an argument similar to the proof of Proposition 2, it can be shown that in any optimal routing, OD pair 1 sends its input traffic along the paths in the clockwise direction of the outer ring, and OD pair 2 sends its input traffic along the paths in the counterclockwise direction of the outer ring. Hence, there are two paths that may be used by OD pair 1 ; the path that traverses the subring $R_{1}$ in the clockwise direction and the path that traverses the subring $R_{1}$ in the counterclockwise direction. The same claim is true for OD pair 2 with subring $R_{1}$ replaced by subring $R_{2}$. Denote the flow along the path of OD pair 1 that goes counterclockwise around ring $R_{1}$ by $x_{1}$ and the flow along the path of OD pair 2 that goes counterclockwise around ring $R_{2}$ by $x_{2}$. Assume that the OD pairs whose origins and destinations are located on one of the subrings use the two paths that consist exclusively of the links of that subring. Denote the flows of the counterclockwise paths of OD pairs 3 and 4 around ring $R_{1}$ by $x_{3}$ and $x_{4}$ respectively, and the flow of the counterclockwise path of OD pair 5 around ring $R_{2}$ by $x_{5}$.

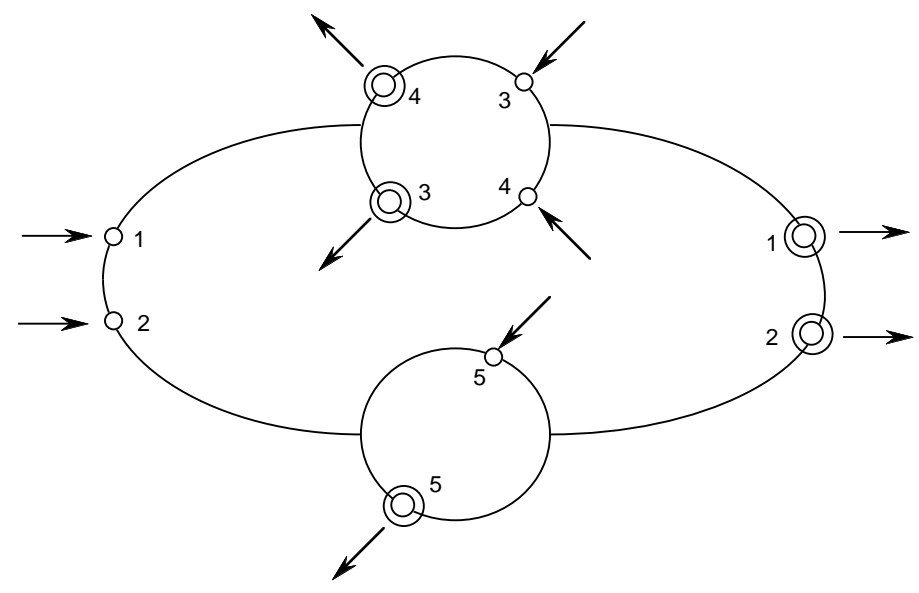

Figure 9. A ring network that includes two subrings and five OD pairs. The circles represent the origins, whereas the double circles represent the destinations.

Let $S_{0}=\left\{x_{1}, x_{2}, x_{3}, x_{4}, x_{5}\right\}$ and solve the relaxed version of problem (F1) for the path flow variables in $S_{0}$. In 
terms of the variables in $S_{0}$, the flows on the links of this network are equal to an integer plus or minus the following quantities:

$$
\begin{gathered}
x_{1}, x_{2}, x_{3}, x_{4}, x_{5}, \\
x_{1}+x_{3}, x_{1}+x_{4}, x_{3}+x_{4}, x_{2}+x_{5},
\end{gathered}
$$

It can be verified analytically or computationally that $x=\left[x_{1}, \ldots, x_{5}\right]=[1 / 2,1 / 2,1 / 2,1 / 2,1 / 2]$ is an optimal solution of the relaxed problem. Since the quantities in the second line of the above collection are integer at the optimal solution $x$, we search for a nonzero vector $d=\left[d_{1}, \ldots, d_{5}\right]$ that satisfies

$$
d_{1}+d_{3}=0, \quad d_{1}+d_{4}=0, \quad d_{3}+d_{4}=0, \quad d_{2}+d_{5}=0
$$

It follows that $d=[0,1,0,0,-1]$ satisfies these equations and therefore is a cost preserving direction. We change $x$ to $x+1 / 2 d=[1 / 2,1,1 / 2,1 / 2,0]$, which is another optimal solution of the relaxed problem, in which OD pairs 2 and 5 send their input traffic completely along a single path. In this new optimal solution, only $x_{1}, x_{3}$, and $x_{4}$ are noninteger, and the flows of the links that belong to paths used by OD pairs 1, 3, and 4 are equal to an integer plus or minus the following quantities:

$$
\begin{gathered}
x_{1}, x_{3}, x_{4}, \\
x_{1}+x_{3}, x_{1}+x_{4}, x_{3}+x_{4} .
\end{gathered}
$$

All the quantities in the second line above are integer at the new optimal solution $x$. However, there exists no nonzero vector $d=\left[d_{1}, d_{3}, d_{4}\right]$ which satisfies

$$
d_{1}+d_{3}=0, \quad d_{1}+d_{4}=0, \quad d_{3}+d_{4}=0
$$

Therefore, we search for some OD pair $i$, where $i=1,3$ or 4 , such that rounding $x_{i}$ to 0 or 1 gives the most favorable cost change. Due to symmetry of the location of OD pairs around subring $R_{1}$ and the symmetry of the link flows at the current solution, it can be seen that setting any one of the $x_{i}$ 's to 0 or 1 yields the same amount of cost change. Therefore, we choose to set $x_{1}$ to 1 and update $x$ to $x=[1,1,1 / 2,1 / 2,0]$. Let $S_{1}=\left\{x_{3}, x_{4}\right\}$ and resolve the relaxed problem for the path flow variables in $S_{1}$, while the remaining variables are fixed at 0 or 1 . It can be verified that $x=[1,1,1,0,0]$ is an optimal solution of this problem, which also turns out to be the optimal solution of problem (F1) for this network.

In all the above examples, the given routing algorithm finds the integer optimal solution. We have not encountered, analytically or computationally, an example for which the algorithm does not yield an integer optimal solution. We believe that such examples do exist, but that they are very rare.

\section{The No Wavelength Conversion Case}

For any network topology, the integer optimal (or slightly suboptimal) solution of problem (F1) obtained at the end of the above algorithm specifies a routing for the OD pairs in which all OD pairs send their input traffic completely along a single path. We call such a routing an integer routing. In some cases, for this routing, a wavelength assignment can be obtained that involves no wavelength conversion at the intermediate nodes. The resulting routing-wavelength assignment specifies an integer optimal (or slightly suboptimal) solution for the no wavelength conversion problem (F2). One such situation was shown in Proposition 4 for ring networks, in which the origins and the destinations can be separated. The idea there was that the integer optimal solution of problem (F1) specifies a routing, in which the paths can be ordered in both clockwise and counterclockwise directions. 
The problem thus reduces to two independent line networks and the wavelength allocation algorithm for the line network, given in the proof of Proposition 1, can be used to find the optimal wavelength assignment with no wavelength conversion. Using the same reasoning, it can be shown that in an arbitrary ring network, given an integer routing, if the corresponding paths "do not wrap around each other" in both directions, a wavelength assignment with no wavelength conversion can be found. For the general case when the paths wrap around in a ring network, we have the following result (see also [WiW98]). We say that a wavelength assignment is valid if along each path, for any successive links that were assigned different wavelengths, there is a wavelength converter at the intermediate node joining these links.

Proposition 5: $\quad$ Suppose that we are given a ring network with multiple OD pairs and an integer routing. Assume that one of the nodes the ring network is equipped with a wavelength converter that has full wavelength conversion capability. Then a valid wavelength assignment can be found for this routing.

Proof: Suppose that the ring is cut at the node with the wavelength converter to form a line network. Paths in the given routing that cross this node (i.e., that wrap around the ring) are broken into two paths. Then the wavelength allocation algorithm for the line network can be used to find a wavelength assignment for these paths. Paths which were broken into two may be assigned two different wavelengths, but these wavelengths are converted using the wavelength converter at this node, showing that the resulting wavelength assignment is valid. Q.E.D.

\section{CONCLUSIONS AND EXTENSIONS}

In this paper, we present several alternative optimal RWA formulations for optical networks having different wavelength conversion capabilities, with the goal of developing efficient methods for computing optimal or nearoptimal RWA policies under realistic assumptions. We propose new integer-linear programming formulations, which tend to have integer optimal solutions even when the integrality constraints are relaxed, thereby allowing the problem to be solved optimally by fast and highly efficient linear (not integer) programming methods. We prove the optimality of the resulting solutions for specific topologies. Our method of proof for these topologies gives rise to a new efficient algorithm that can be used for arbitrary networks with full wavelength conversion capability to find an optimal or near-optimal integer routing solution. Given this integer routing solution, we present an approach that finds a valid wavelength assignment for a ring network, provided that there is one wavelength converter at any of the nodes of the ring. An interesting research problem is to come up with an efficient method of placing wavelength converters at some of the nodes of a given arbitrary network, so that the same approach could be used to find a valid wavelength assignment for any integer routing.

We also propose various exact penalty formulations of the problem that can be used within several network design contexts. In particular, these formulations can be used to determine the number of wavelengths required on each link and the placement of wavelength converters in order to support the input traffic of a given set of OD pairs. Exact penalty formulations can also be used for reconfiguration purposes, to adapt the current routing and wavelength assignment scheme to changes in the network topology and traffic patterns.

\section{REFERENCES}


[Ber98] Bertsekas, D. P., Network Optimization: Continuous and Discrete Models, Athena Scientific, Belmont, MA, 1998.

[Ber99] Bertsekas, D. P., Nonlinear Programming, 2nd edition, Athena Scientific, Belmont, MA, 1999.

[Ber00] Bertsekas, D. P., Dynamic Programming and Optimal Control, Vol.I, Athena Scientific, Belmont, MA, 2000.

[BeG92] Bertsekas, D. P., and Gallager, R. G., Data Networks, 2nd edition, Prentice-Hall, Englewood Cliffs, NJ, 1992 .

[BaH96] Barry, R., and Humblet, P., "Models of Blocking Probability in All-Optical Networks with and without Wavelength Changers," IEEE J. Select. Areas Commun., vol. 14, no.5, pp. 858-867, June 1996.

[BaM96] Banerjee, D., and Mukherjee, B., "A Practical Approach for Routing and Wavelength Assignment in Large Wavelength-Routed Optical Networks," IEEE J. Select. Areas Commun., vol. 14, no.5, pp. 903-908, June 1996.

[CGK92] Chlamtac, I., Ganz, A., and Karmi, G., "Lightpath Communications: An Approach to High-Bandwidth Optical WAN's," IEEE Trans. Commun., vol. 40, pp. 1171-1182, July 1992.

[GSKR99] Gerstel, O., Sasaki, G., Kutten S., and Ramaswami, R., "Worst-Case Analysis of Dynamic Wavelength Allocation in Optical Networks," IEEE/ACM Transactions on Networking, vol. 7, no. 6, pp. 833-845, Dec. 1999.

[GeK97] Gerstel, O., and Kutten S., "Dynamic Wavelength Allocation in All-Optical Ring Networks," in Proc. ICC '97, pp. 432-436, 1997.

[KoA96] Kovacevic, M., and Acampora A., "Benefits of Wavelength Translation in All-Optical Clear-Channel Networks," IEEE J. Select. Areas Commun., vol. 14, pp. 868-880, 1996.

[MoA98] Mokhtar, A., and Azizoglu, M., "Adaptive Wavelength Routing in All-Optical Networks," IEEE/ACM Transactions on Networking, vol. 6, pp. 197-206, 1998.

[MBRM96] Mukherjee, B., Banerjee, D., Ramamurthy, S., and Mukherjee, A., "Some Principles for Designing a Wide-Area WDM Optical Network," IEEE/ACM Transactions on Networking, vol. 4, pp. 684-696, 1996.

[McS92] McEliece, R. J., and Sivarajan, K. N., "Maximizing Marginal Revenue in Generalized Blocking Service Networks," in Proc. 30th Annual Allerton Conf. Commun. Contr. Comput., vol. 18, pp. 455-464, 1992.

[RaS95] Ramaswami, R., and Sivarajan, K. N., "Routing and Wavelength Assignment in All-Optical Networks," IEEE/ACM Transactions on Networking, vol. 3, pp. 489-499, Oct. 1995.

[RaS98] Ramaswami, R., and Sasaki, G., "Multiwavelength Optical Networks with Limited Wavelength Conversion," IEEE/ACM Transactions on Networking, vol. 6, no. 6, pp. 744-754, Dec. 1998.

[RaSi98] Ramaswami, R., and Sivarajan, K. N., "Optical Networks: A Practical Perspective," CA: Morgan Kaufmann, 1998.

[SAS96] Subramaniam, S., Azizoglu, M., and Somani, A. K., "All-Optical Networks with Sparse Wavelength 
Conversion," IEEE/ACM Transactions on Networking, vol. 4, no. 4, pp. 544-557, June 1996.

[SuB97] Subramaniam, S., and Richard, A. B., "Wavelength Assignment in Fixed Routing WDM Networks," in Proc. ICC '97, pp. 406-410.

[Tuc75] Tucker, A., "Coloring a family of circular arcs," SIAM J. on Applied Math, vol. 29, no. 3, pp. 493-502, 1975.

[WiW98] Wilfong, G., and Winkler, P., "Ring routing and wavelength translation," Proc. Symp. on Discrete Algorithms, 1998, pp. 333-341. 